\title{
Asymptotic Behavior of Ground State Radial Solutions for $p$-Laplacian Problems
}

\author{
Sonia Ben Othman, ${ }^{1}$ Rym Chemmam, ${ }^{1}$ and Habib Mâagli ${ }^{2}$ \\ ${ }^{1}$ Département de Mathématiques, Faculté des Sciences de Tunis, Campus Universitaire, 2092 Tunis, Tunisia \\ ${ }^{2}$ King Abdulaziz University, College of Sciences and Arts, Rabigh Campus, Department of Mathematics, P.O. Box 344, \\ Rabigh 21911, Saudi Arabia
}

Correspondence should be addressed to Sonia Ben Othman; sonia.benothman@fsb.rnu.tn

Received 13 September 2012; Accepted 28 December 2012

Academic Editor: Francisco B. Gallego

Copyright (C) 2013 Sonia Ben Othman et al. This is an open access article distributed under the Creative Commons Attribution License, which permits unrestricted use, distribution, and reproduction in any medium, provided the original work is properly cited.

Let $p>1$, we take up the existence, the uniqueness and the asymptotic behavior of a positive continuous solution to the following nonlinear problem in $(0,+\infty),(1 / A)\left(A \phi_{p}\left(u^{\prime}\right)\right)+q(x) u^{\alpha}=0, \lim _{x \rightarrow 0} A \phi_{p}\left(u^{\prime}\right)(x)=0, \lim _{x \rightarrow+\infty} u(x)=0$, where $\alpha<p-1$, $\phi_{p}(t)=t|t|^{p-2}(t \in \mathbb{R}), A$ is a positive differentiable function in $(0,+\infty)$ and $q$ is a positive continuous function in $(0,+\infty)$ such that there exists $c>0$ satisfying for each $x$ in $(0,+\infty), 1 / c \leq q(x)(1+x)^{\beta} \exp \left(-\int_{1}^{x+1}(z(s) / s) d s\right) \leq c, \beta \geq p$ and $z \in C([1,+\infty))$ such that $\lim _{t \rightarrow+\infty} z(t)=0$.

\section{Introduction}

Let $p>1$ and $\alpha<p-1$. The following differential equation:

$$
\begin{aligned}
L_{p} u:=\frac{1}{A}\left(A \Phi_{p}\left(u^{\prime}\right)\right)^{\prime}= & -q(x) u^{\alpha}, \\
& \text { in }(0, \omega), \quad \omega \in(0,+\infty]
\end{aligned}
$$

has been studied with various boundary conditions, where $A$ is a continuous function in $[0,+\infty)$, differentiable and positive in $(0,+\infty), q$ is a nonnegative continuous function in $(0,+\infty)$, and $\Phi_{p}(t)=t|t|^{p-2},(t \in \mathbb{R})$ (see [1-15]).

For a function $u$ depending only on $t=|x|, x \in \mathbb{R}^{n}$, and $A(t)=t^{n-1}$, the operator $L_{p}$ is the radially symmetric $p$-Laplacian $\Delta_{p} u=\operatorname{div}\left(|\nabla u|^{p-2} \nabla u\right)$ in $\mathbb{R}^{n}$ and the equation of type (1) arises also as radial solutions of the Monge-Ampère equation (see [13]).
In this paper, our main purpose is to obtain the existence of a unique positive solution to the following boundary value problem:

$$
\begin{aligned}
& \frac{1}{A}\left(A \Phi_{p}\left(u^{\prime}\right)\right)^{\prime}+q(x) u^{\alpha}=0, \quad \text { in }(0, \infty), \\
& A \Phi_{p}\left(u^{\prime}\right)(0):=\lim _{x \rightarrow 0^{+}} A \Phi_{p}\left(u^{\prime}\right)(x)=0, \\
& \lim _{x \rightarrow+\infty} u(x)=0,
\end{aligned}
$$

and to establish estimates on such solution under an appropriate condition on $q$.

The study of this type of $(1)$ is motivated by $[5,15]$. Namely, in the special case $A(t)=t^{n-1} g(t), g \in C^{1}\left(\mathbb{R}^{+}\right)$and $f(u)=u^{\alpha}, \alpha>-1$, the authors in [15] studied (1) and gave some uniqueness results. In this work, we consider a wider class of weights $A$ and we aim to extend the study of (1) in [15], to $\alpha<p-1$. 
For the case $\alpha<0$, the problem $(P)$ has been studied in [5]. Indeed, the authors of [5] proved the following existence result.

Theorem 1. The problem $(P)$ has a unique positive solution $u \in C([0,+\infty))$ satisfying for each $x \in(0,+\infty)$

$$
\frac{1}{c} G_{p} q(x) \leq u(x) \leq c\left(G_{p} q(x)\right)^{(p-1) /(p-1-\alpha)},
$$

where $c$ is a positive constant and $G_{p} q$ is the function defined on $(0,+\infty)$ by

$$
G_{p} q(x):=\int_{x}^{+\infty}\left(\frac{1}{A(t)} \int_{0}^{t} A(s) q(s) d s\right)^{1 /(p-1)} d t
$$

We shall improve in this paper the above asymptotic behavior of the solution of problem $(P)$ and we extend the study of $(P)$ to $0 \leq \alpha<p-1$.

The pure elliptic problem of type

$$
\begin{gathered}
-\Delta u+q(x) u^{\alpha}=0, \quad x \in \mathbb{R}^{n}(n \geq 3), \alpha<1, \\
u>0 \text { in } \mathbb{R}^{n}, \\
\lim _{|x| \rightarrow+\infty} u(x)=0
\end{gathered}
$$

has been investigated by several authors with zero Dirichlet boundary value; we refer the reader to [16-24] and the references therein. More recently, applying Karamata regular variation theory, Chemmam et al. gave in [17] the asymptotic behavior of solutions of problem (Q). In this work, we aim to extend the result established in [17] to the radial case associated to problem $(P)$.

To simplify our statements, we need to fix some notations and make some assumptions. Throughout this paper, we shall use $\mathscr{K}$, to denote the set of Karamata functions $L$ defined on $[1,+\infty)$ by

$$
L(t):=c \exp \left(\int_{1}^{t} \frac{z(s)}{s} d s\right)
$$

where $c$ is a positive constant and $z \in C([1, \infty[)$ such that $\lim _{t \rightarrow+\infty} z(t)=0$.

It is clear that $L \in \mathscr{K}$ if and only if $L$ is a positive function in $C^{1}([1, \infty[)$ such that

$$
\lim _{t \rightarrow \infty} \frac{t L^{\prime}(t)}{L(t)}=0
$$

For two nonnegative functions $f$ and $g$ on a set $S$, we write $f(x) \approx g(x), x \in S$, if there exists a constant $c>0$ such that $(1 / c) g(x) \leq f(x) \leq c g(x)$, for each $x \in S$.

The letter $c$ will denote a generic positive constant which may vary from line to line.

Furthermore, we point out that if $f$ is a nonnegative continuous function in $\left[0,+\infty\left[\right.\right.$, then the function $G_{p} f$ defined on $(0,+\infty)$ by

$$
G_{p} f(x):=\int_{x}^{+\infty}\left(\frac{1}{A(t)} \int_{0}^{t} A(s) f(s) d s\right)^{1 /(p-1)} d t
$$

is the solution of the problem

$$
\begin{gathered}
-\frac{1}{A}\left(A \Phi_{p}\left(u^{\prime}\right)\right)^{\prime}=f, \quad \operatorname{in}(0,+\infty), \\
A \Phi_{p}\left(u^{\prime}\right)(0)=0, \quad \lim _{x \rightarrow+\infty} u(x)=0 .
\end{gathered}
$$

As it is mentioned above, our main purpose in this paper is to establish existence and global behavior of a positive solution of problem $(P)$. Let us introduce our hypotheses.

Here, the function $A$ is continuous in $[0,+\infty)$, differentiable and positive in $(0,+\infty)$ such that

$$
A(x) \approx x^{\lambda}
$$

with $\lambda>p-1$.

The function $q$ is required to satisfy the following hypothesis. that

$(H) q$ is a positive measurable function on $(0,+\infty)$ such

$$
q(x) \approx \frac{L(1+x)}{(1+x)^{\beta}},
$$

with $\beta \geq p$ and the function $L \in \mathscr{K}$ such that $\int_{1}^{+\infty} t^{(1-\beta) /(p-1)}(L(t))^{1 /(p-1)} d t<+\infty$.

Remark 2. We need to verify condition

$$
\int_{1}^{+\infty} t^{(1-\beta) /(p-1)}(L(t))^{1 /(p-1)} d t<+\infty
$$

in hypothesis $(H)$, only if $\beta=p$, (see Lemma 6 below).

As a typical example of function $q$ satisfying $(H)$, we quote the following.

Example 3. Put $q(x):=(1+x)^{-\beta}(\log (x+2))^{-v}, x \in(0,+\infty)$. Then for $\beta>p$ and $\nu \in \mathbb{R}$ or $\beta=p$ and $\nu>p-1$, the function $q$ satisfies $(H)$.

Now, we are ready to state our main result.

Theorem 4. Assume $(H)$. Then problem $(P)$ has a unique positive continuous solution $u$ satisfying for $x \in(0,+\infty)$,

$$
u(x) \approx \theta_{\beta}(x),
$$


where $\theta_{\beta}$ is the function defined on $[0,+\infty)$ by

$$
\theta_{\beta}(x):=\left\{\begin{array}{l}
\left(\int_{x+1}^{\infty} \frac{(L(s))^{1 /(p-1)}}{s} d s\right)^{(p-1) /(p-1-\alpha)} \\
\text { if } \beta=p, \\
\frac{(L(1+x))^{1 /(p-1-\alpha)}}{(1+x)^{(\beta-p) /(p-1-\alpha)}} \\
\text { if } p<\beta<\frac{(\lambda+1)(p-1-\alpha)+\alpha p}{p-1}, \\
(1+x)^{(p-1-\lambda) /(p-1)}, \\
\text { if } \beta>\frac{(\lambda+1)(p-1-\alpha)+\alpha p}{p-1}, \\
(1+x)^{(p-1-\lambda) /(p-1)\left(\int_{1}^{x+2} \frac{L(s)}{s} d s\right)^{1 /(p-1-\alpha)}} \\
\text { if } \beta=\frac{(\lambda+1)(p-1-\alpha)+\alpha p}{p-1} .
\end{array}\right.
$$

The main body of the paper is organized as follows. In Section 2, we establish some estimates and we recall some known results on functions belonging to $\mathscr{K}$. Theorem 4 is proved in Section 3. The last Section is reserved to some applications.

\section{Key Estimates}

In what follows, we are going to give estimates on the functions $G_{p} q$ and $G_{p}\left(q \theta_{\beta}^{\alpha}\right)$, where $q$ is a function satisfying $(H)$ and $\theta_{\beta}$ is the function given by (12). First, we recall some fundamental properties of functions belonging to the class $\mathscr{K}$, taken from $[17,22]$.

Lemma 5. Let $L_{1}, L_{2} \in \mathscr{K}, m \in \mathbb{R}$ and $\varepsilon>0$. Then one has $L_{1} L_{2} \in \mathscr{K}, L_{1}^{m} \in \mathscr{K}$ and $\lim _{t \rightarrow+\infty} t^{-\varepsilon} L_{1}(t)=0$.

Lemma 6 (Karamata's theorem). Let $\mu \in \mathbb{R}$ and $L$ be a function in $\mathscr{K}$. Then one has the following properties:

(i) If $\mu<-1$, then $\int_{1}^{\infty} s^{\mu} L(s) d s$ converges and

$$
\int_{t}^{\infty} s^{\mu} L(s) d s \underset{t \rightarrow \infty}{\sim} \frac{-t^{1+\mu} L(t)}{\mu+1} .
$$

(ii) If $\mu>-1$, then $\int_{1}^{\infty} s^{\mu} L(s) d s$ diverges and

$$
\int_{1}^{t} s^{\mu} L(s) d s \underset{t \rightarrow \infty}{\sim} \frac{t^{1+\mu} L(t)}{\mu+1} .
$$

Lemma 7. Let $L \in \mathscr{K}$. Then there exists $m>0$ such that for $t \geq 1$ and $c>0$

$$
\frac{1}{(1+c)^{m}} L(t) \leq L(c+t) \leq(1+c)^{m} L(t) .
$$

Lemma 8. Let $L \in \mathscr{K}$, then one has

$$
t \longrightarrow \int_{1}^{t+1} \frac{L(s)}{s} d s \in \mathscr{K} .
$$

If further $\int_{1}^{\infty}(L(s) / s) d s$ converges, one has

$$
t \longrightarrow \int_{t}^{\infty} \frac{L(s)}{s} d s \in \mathscr{K} .
$$

Now, we are able to prove the following propositions which play a crucial role in this paper.

Proposition 9. Let $q$ be a function satisfying $(H)$. Then one has for $x \in(0, \infty)$

$$
G_{p} q(x) \approx \Psi(x),
$$

where $\psi$ is the function defined by

$$
\Psi(x):=\left\{\begin{array}{l}
\int_{x+1}^{\infty} \frac{(L(s))^{1 /(p-1)}}{s} d s, \\
\text { if } \beta=p, \\
\frac{(L(x+1))^{1 /(p-1)}}{(x+1)^{(\beta-p) /(p-1)},} \\
\text { if } p<\beta<\lambda+1, \\
(x+1)^{(p-1-\lambda) /(p-1)}\left(\int_{1}^{x+2} \frac{L(s)}{s} d s\right)^{1 /(p-1)}, \\
\text { if } \beta=\lambda+1, \\
(x+1)^{(p-1-\lambda) /(p-1)}, \\
\text { if } \beta>\lambda+1 .
\end{array}\right.
$$

Proof. For $x \in(0, \infty)$, we have

$$
G_{p} q(x) \approx \int_{x}^{\infty} t^{\lambda /(1-p)}\left(\int_{0}^{t} s^{\lambda}(1+s)^{-\beta} L(1+s) d s\right)^{1 /(p-1)} d t .
$$

Put

$$
\begin{array}{r}
h(x)=: \int_{x}^{\infty} t^{\lambda /(1-p)}\left(\int_{0}^{t} s^{\lambda}(1+s)^{-\beta} L(1+s) d s\right)^{1 /(p-1)} d t \\
x \in(0, \infty)
\end{array}
$$

To prove the result, it is sufficient to show that $h(x) \approx \Psi(x)$ for $x \in(0, \infty)$.

Since the function $h$ is continuous and positive in $[0,1]$, we have

$$
h(x) \approx 1, \quad x \in[0,1] .
$$

Now, assume that $x \in[1, \infty)$, then we have

$$
\begin{aligned}
h(x)=\int_{x}^{\infty} t^{\lambda /(1-p)}\left(\int_{0}^{1} s^{\lambda}(1+s)^{-\beta} L(1+s) d s\right. & \\
& \left.+\int_{1}^{t} s^{\lambda}(1+s)^{-\beta} L(1+s) d s\right)^{1 /(p-1)} d t .
\end{aligned}
$$

It follows from Lemma 7 that

$$
h(x) \approx \int_{x}^{\infty} t^{\lambda /(1-p)}\left(1+\int_{1}^{t} s^{\lambda-\beta} L(s) d s\right)^{1 /(p-1)} d t .
$$


To reach our estimates, we consider the following cases.

(i) If $p<\beta<\lambda+1$, then it follows from Lemma 6 that

$$
h(x) \approx \int_{x}^{\infty} t^{(1-\beta) /(p-1)} L(t)^{1 /(p-1)} d t
$$

Now, using Lemma 5 and again Lemma 6, we deduce that

$$
h(x) \approx \frac{(L(x))^{1 /(p-1)}}{x^{(\beta-p) /(p-1)}}
$$

(ii) If $\beta>\lambda+1$, then it follows from Lemma 6 , that $\int_{1}^{\infty} s^{\lambda-\beta} L(s) d s<\infty$. So, since $\lambda>p-1$, we have

$$
h(x) \approx x^{(p-\lambda-1) /(p-1)}
$$

(iii) If $\beta=\lambda+1$, then for each $t \in[x,+\infty)$, we have

$$
\left(1+\int_{1}^{t} \frac{L(s)}{s} d s\right)^{1 /(p-1)} \approx\left(\int_{1}^{t+1} \frac{L(s)}{s} d s\right)^{1 /(p-1)} .
$$

Since the function $t \mapsto\left(\int_{1}^{t+1}(L(s) / s) d s\right)^{1 /(p-1)}$ is in $\mathscr{K}$, then using the fact that $\lambda>p-1$ and Lemma 6 , we obtain that

$$
h(x) \approx x^{(p-1-\lambda) /(p-1)}\left(\int_{1}^{x+1} \frac{L(s)}{s} d s\right)^{1 /(p-1)} .
$$

(iv) If $\beta=p$, we have by Lemma 6 that

$$
\int_{1}^{t} s^{\lambda-p} L(s) d s \approx t^{\lambda-p+1} L(t)
$$

this yields to

$$
h(x) \approx \int_{x}^{\infty} \frac{L^{1 /(p-1)}(t)}{t} d t
$$

Hence, we reach the result by combining (22) with the estimates stated in each case above. This completes the proof.

Proposition 10. Let $q$ be a function satisfying $(H)$ and let $\theta_{\beta}$ be the function given by (12). Then for $x \in(0, \infty)$, one has

$$
G_{p}\left(q \theta_{\beta}^{\alpha}\right)(x) \approx \theta_{\beta}(x)
$$

Proof. Let $\beta \geq p$ and $\lambda>p-1$, we obtain by simple calculus that for $x \in(0, \infty)$,

$$
q(x) \theta_{\beta}^{\alpha}(x) \approx \widetilde{q}(x),
$$

where

$$
\tilde{q}(x)=:\left\{\begin{array}{l}
\frac{L(1+x)}{(1+x)^{p}}\left(\int_{x+1}^{\infty} \frac{(L(s))^{1 /(p-1)}}{s} d s\right)^{\alpha(p-1) /(p-1-\alpha)} \\
\text { if } \beta=p \\
\frac{(L(1+x))^{(p-1) /(p-1-\alpha)}}{(1+x)^{(\beta-\alpha(p-\beta) /(p-1-\alpha))}} \\
\text { if } p<\beta<\frac{(\lambda+1)(p-1-\alpha)+\alpha p}{p-1}, \\
\frac{L(1+x)}{(1+x)^{(\beta-\alpha(p-1-\lambda) /(p-1))}} \\
\text { if } \beta>\frac{(\lambda+1)(p-1-\alpha)+\alpha p}{p-1}, \\
\frac{L(1+x)\left(\int_{1}^{x+2} \frac{L(s)}{s} d s\right)}{(1+x)}\left(\int_{1}^{\alpha / 1}\right) \\
\text { if } \beta=\frac{(\lambda+1)(p-1-\alpha)+\alpha p}{p-1} .
\end{array}\right.
$$

So, one can see that

$$
\widetilde{q}(x)=(1+x)^{-\delta} \widetilde{L}(1+x),
$$

where $\delta \geq p$. Then, using Lemmas 5,7 , and 8 , we obtain that $\widetilde{L} \in \mathscr{K}$ and $\int_{1}^{\infty} t^{(1-\delta) /(p-1)}(\widetilde{L}(t))^{1 /(p-1)} d t<+\infty$. Hence, it follows from Proposition 9 that

$$
G_{p}\left(q \theta_{\beta}^{\alpha}\right)(x) \approx G_{p} \tilde{q}(x) \approx \tilde{\psi}(x), \quad x \in(0, \infty),
$$

where $\widetilde{\psi}$ is the function defined in (19) by replacing $L$ by $\widetilde{L}$ and $\beta$ by $\delta$. This ends the proof.

\section{Proof of Theorem 4}

3.1. Existence and Asymptotic Behavior. Let $q$ be a function satisfying $(H)$ and let $\theta_{\beta}$ be the function given by (12). By Proposition 10, there exists a constant $m \geq 1$ such that for each $x \in(0, \infty)$

$$
\frac{1}{m} \theta_{\beta}(x) \leq G_{p}\left(q \theta_{\beta}^{\alpha}\right)(x) \leq m \theta_{\beta}(x) .
$$

We look now at the existence of positive solution of problem $(P)$ satisfying $(11)$.

For the case $\alpha<0$, the existence of a positive continuous solution to problem $(P)$ is due to [5]. Now, we look to the existence result of problem $(P)$ when $0 \leq \alpha<p-1$ and we give precise asymptotic behavior of such solution for $\alpha<$ $p-1$. For that, we split the proof into two cases.

Case $1(\alpha<0)$. Let $u$ be a positive continuous solution of problem $(P)$. So, in order to obtain estimates (11) on the function $u$, we need the following comparison result. 
Lemma 11. Let $\alpha<0$ and $u_{1}, u_{2} \in C^{1}((0, \infty)) \cap C([0, \infty))$ such that

$$
\begin{gathered}
-L_{p} u_{1}(x) \leq q(x) u_{1}^{\alpha}, \quad \text { in }(0, \infty), \\
A \Phi_{p}\left(u_{1}^{\prime}\right)(0)=0, \quad \lim _{x \rightarrow+\infty} u_{1}(x)=0, \\
-L_{p} u_{2}(x) \geq q(x) u_{2}^{\alpha}, \quad \text { in }(0, \infty), \\
A \Phi_{p}\left(u_{2}^{\prime}\right)(0)=0, \quad \lim _{x \rightarrow+\infty} u_{2}(x)=0 .
\end{gathered}
$$

Then $u_{1} \leq u_{2}$.

Proof. Suppose that $u_{1}\left(x_{0}\right)>u_{2}\left(x_{0}\right)$ for some $x_{0} \in(0, \infty)$. Then there exists $x_{1}, x_{2} \in[0, \infty]$, such that $0 \leq x_{1}<x_{0}<$ $x_{2} \leq \infty$ and for $x_{1}<x<x_{2}, u_{1}(x)>u_{2}(x)$ with $u_{1}\left(x_{2}\right)=$ $u_{2}\left(x_{2}\right), u_{1}\left(x_{1}\right)=u_{2}\left(x_{1}\right)$ or $x_{1}=0$.

By an elementary argument, we have

$$
A \Phi_{p}\left(u_{2}^{\prime}\right)\left(x_{1}\right) \leq A \Phi_{p}\left(u_{1}^{\prime}\right)\left(x_{1}\right) .
$$

On the other hand, since $\alpha<0$, then $u_{1}^{\alpha}(x)<u_{2}^{\alpha}(x)$, for each $x \in\left(x_{1}, x_{2}\right)$. This yields to

$$
L_{p} u_{1}-L_{p} u_{2} \geq q\left(u_{2}^{\alpha}-u_{1}^{\alpha}\right) \geq 0, \quad \text { on }\left(x_{1}, x_{2}\right) .
$$

Using further (40), we deduce that the function $\omega(x)$ := $\left(A \Phi_{p}\left(u_{1}^{\prime}\right)-A \Phi_{p}\left(u_{2}^{\prime}\right)\right)(x)$ is nondecreasing on $\left(x_{1}, x_{2}\right)$ with $\omega\left(x_{1}\right) \geq 0$. Hence, from the monotonicity of $\Phi_{p}$, we obtain that the function $x \mapsto\left(u_{1}-u_{2}\right)(x)$ is nondecreasing on $\left(x_{1}, x_{2}\right)$ with $\left(u_{1}-u_{2}\right)\left(x_{1}\right) \geq 0$ and $\left(u_{1}-u_{2}\right)\left(x_{2}\right)=0$. This yields to a contradiction, which completes the proof.

Now, we are ready to prove (11). Put $c=m^{-\alpha /(p-1-\alpha)}$ and $v:=G_{p}\left(q \theta_{\beta}^{\alpha}\right)$. It follows from (7) that the function $v$ satisfies

$$
-L_{p} v=q \theta_{\beta}^{\alpha}, \quad \text { in }(0, \infty) .
$$

According to (37), we obtain by simple calculation that $(1 / c) v$ and $c v$ satisfy, respectively, (38) and (39). Thus, we deduce by Lemma 11 that

$$
\frac{1}{c} v(x) \leq u(x) \leq c v(x), \quad x \in(0, \infty) .
$$

This implies (11) by using (37).

Case $2(0 \leq \alpha<p-1)$. Put $c_{0}=m^{(p-1) /(p-1-\alpha)}$ and let

$$
\Lambda:=\left\{u \in C((0, \infty)) ; \frac{1}{c_{0}} \theta_{\beta} \leq u \leq c_{0} \theta_{\beta}\right\} .
$$

Obviously, the function $\theta_{\beta}$ belongs to $C((0, \infty))$ and so $\Lambda$ is not empty. We consider the integral operator $T$ on $\Lambda$ defined by

$$
T u(x):=G_{p}\left(q u^{\alpha}\right)(x), \quad x \in(0, \infty) .
$$

We shall prove that $T$ has a fixed point in $\Lambda$, to construct a solution of problem $(P)$. For this aim, we look at first that $T \Lambda \subset \Lambda$. Let $u \in \Lambda$, then we have for each $x \in(0, \infty)$

$$
\frac{1}{c_{0}^{\alpha}}\left(q \theta_{\beta}^{\alpha}\right)(x) \leq q(x) u^{\alpha}(x) \leq c_{0}^{\alpha}\left(q \theta_{\beta}^{\alpha}\right)(x) .
$$

This together with (37) implies that

$$
\frac{1}{m c_{0}^{\alpha /(p-1)}} \theta_{\beta} \leq T u \leq m c_{0}^{\alpha /(p-1)} \theta_{\beta} .
$$

Since $m c_{0}^{\alpha /(p-1)}=c_{0}$ and $T \Lambda \subset C((0, \infty))$, then $T$ leaves invariant the convex $\Lambda$. Moreover, since $\alpha \geq 0$, then the operator $T$ is nondecreasing on $\Lambda$. Now, let $\left\{u_{k}\right\}_{k}$ be the sequence of functions in $C([0,+\infty))$ defined by

$$
u_{0}=\frac{1}{c_{0}} \theta_{\beta}, \quad u_{k+1}=T u_{k}, \quad \text { for } k \in \mathbb{N} .
$$

Since $T \Lambda \subset \Lambda$, we deduce from the monotonicity of $T$ that for $k \in \mathbb{N}$, we have

$$
u_{0} \leq u_{1} \leq \cdots \leq u_{k} \leq u_{k+1} \leq c_{0} \theta_{\beta} .
$$

Thanks to the monotone convergence theorem, we deduce that the sequence $\left\{u_{k}\right\}_{k}$ converges to a function $u \in \Lambda$ which satisfies

$$
u(x)=G_{p}\left(q u^{\alpha}\right)(x), \quad x \in(0, \infty) .
$$

We conclude that $u$ is a positive continuous solution of problem $(P)$ satisfying $(11)$.

3.2. Uniqueness. Assume that $q$ satisfies $(H)$. For $\alpha<0$, the uniqueness of solution to problem $(P)$ follows from Lemma 11 . Thus in the following, we look at the case $0 \leq \alpha<p-1$. Let

$$
\Gamma=\left\{u \in C((0, \infty)): u(x) \approx \theta_{\beta}(x)\right\} .
$$

Let $u$ and $v$ be two positives solutions of problem $(P)$ in $\Gamma$. Then there exists a constant $k \geq 1$ such that

$$
\frac{1}{k} \leq \frac{v}{u} \leq k
$$

This implies that the set

$$
J=\left\{t \in(1,+\infty), \frac{1}{t} u \leq v \leq t u\right\}
$$

is not empty. Now, put $c:=\inf J$, then we aim to show that $c=1$.

Suppose that $c>1$, then we have

$$
\begin{gathered}
-L_{p} v+L_{p}\left(c^{-\alpha /(p-1)} u\right)=q(x)\left(v^{\alpha}-c^{-\alpha} u^{\alpha}\right), \quad \text { in }(0, \infty), \\
\lim _{x \rightarrow 0^{+}}\left(A \Phi_{p}\left(v^{\prime}\right)-A \Phi_{p}\left(c^{-\alpha /(p-1)} u^{\prime}\right)\right)(x)=0, \\
\lim _{x \rightarrow+\infty}\left(v-c^{-\alpha /(p-1)} u\right)(x)=0 .
\end{gathered}
$$


So, we have $-L_{p} v+L_{p}\left(c^{-\alpha /(p-1)} u\right) \geq 0$ in $(0, \infty)$, which implies that the function

$$
\theta(x):=\left(A \Phi_{p}\left(c^{-\alpha /(p-1)} u^{\prime}\right)-A \Phi_{p}\left(v^{\prime}\right)\right)(x)
$$

is nondecreasing on $(0,+\infty)$ with $\lim _{x \rightarrow 0^{+}} \theta(x)=0$. Hence from the monotonicity of $\Phi_{p}$, we obtain that the function $x \mapsto\left(c^{-\alpha /(p-1)} u-v\right)(x)$ is nondecreasing on $[0,+\infty)$ with $\lim _{x \rightarrow \infty}\left(c^{-\alpha /(p-1)} u-v\right)(x)=0$. This implies that $c^{-\alpha /(p-1)} u \leq$ $v$. On the other hand, we deduce by symmetry that $v \leq$ $c^{\alpha /(p-1)} u$. Hence $c^{\alpha /(p-1)} \in J$. Now, since $\alpha<p-1$ and $c>1$, we have $c^{\alpha /(p-1)}<c$. This yields to a contradiction with the fact that $c:=\inf J$. Hence, $c=1$ and then $u=v$.

\section{Applications}

4.1. First Application. Let $q$ be a positive measurable function in $(0, \infty)$ satisfying for $x \in(0, \infty)$

$$
q(x) \approx \frac{(\log (2+x))^{-\sigma}}{(1+x)^{\beta}},
$$

where the real numbers $\beta$ and $\sigma$ satisfy one of the following two conditions:

(i) $\beta>p$ and $\sigma \in \mathbb{R}$,

(ii) $\beta=p$ and $\sigma>p-1$.

Using Theorem 4 , we deduce that problem $(P)$ has a positive continuous solution $u$ in $(0, \infty)$ satisfying the following.

(i) If $\beta>((\lambda+1)(p-1-\alpha)+\alpha p) /(p-1)$, then for $x \in(0, \infty)$

$$
u(x) \approx \frac{1}{(1+x)^{(\lambda-p+1) /(p-1)}} .
$$

(ii) If $\beta=((\lambda+1)(p-1-\alpha)+\alpha p) /(p-1)$ and $\sigma=1$, then for $x \in(0, \infty)$

$u(x) \approx \frac{1}{(1+x)^{(\lambda-p+1) /(p-1)}}(\log \log (3+x))^{1 /(p-1-\alpha)}$.

(iii) If $\beta=((\lambda+1)(p-1-\alpha)+\alpha p) /(p-1)$ and $\sigma<1$, then for $x \in(0, \infty)$

$u(x) \approx \frac{1}{(1+x)^{(\lambda-p+1) /(p-1)}}\left(\log \frac{3}{1+x}\right)^{(1-\sigma) /(p-1-\alpha)}$.

(iv) If $\beta=((\mu+1)(p-1-\alpha)+\alpha p) /(p-1)$ and $\sigma>1$, then for $x \in(0, \infty)$

$$
u(x) \approx \frac{1}{(1+x)^{(\lambda-p+1) /(p-1)}} .
$$

(v) If $p<\beta<((\lambda+1)(p-1-\alpha)+\alpha p) /(p-1)$, then for $x \in(0, \infty)$

$u(x) \approx \frac{1}{(1+x)^{(\beta-p) /(p-1-\alpha)}}(\log (2+x))^{-\sigma /(p-1-\alpha)}$.

(vi) If $\beta=p$ and $\sigma>p-1$, then for $x \in(0, \infty)$

$$
u(x) \approx(\log (2+x))^{(p-1-\sigma) /(p-1-\alpha)} .
$$

4.2. Second Application. Let $q$ be a function satisfying $(H)$ and let $\alpha, \gamma<p-1$. We are interested in the following nonlinear problem:

$$
\begin{gathered}
-\frac{1}{A}\left(A \Phi_{p}\left(u^{\prime}\right)\right)^{\prime}+\frac{\gamma}{u} \Phi_{p}\left(u^{\prime}\right) u^{\prime}=q(x) u^{\alpha}, \quad \text { in }(0, \infty), \\
A \Phi_{p}\left(u^{\prime}\right)(0)=0, \quad \lim _{x \rightarrow \infty} u(x)=0 .
\end{gathered}
$$

Put $v=u^{1-\gamma /(p-1)}$, then by a simple calculus, we obtain that $v$ satisfies

$$
\begin{gathered}
-\frac{1}{A}\left(A \Phi_{p}\left(v^{\prime}\right)\right)^{\prime}=\left(\frac{p-1-\gamma}{p-1}\right)^{p-1} q(x) v^{(\alpha-\gamma)(p-1) /(p-1-\gamma),} \\
\text { in }(0, \infty), \\
A \Phi_{p}\left(v^{\prime}\right)(0)=0, \quad \lim _{x \rightarrow \infty} v(x)=0 .
\end{gathered}
$$

Using Theorem 4, we deduce that problem (64) has a unique solution $v$ such that

$$
\begin{aligned}
& v(x) \approx \widetilde{\theta}_{\beta}(x) \\
& :=\left\{\begin{array}{l}
\left(\int_{x+1}^{\infty} \frac{(L(s))^{1 /(p-1)}}{s} d s\right)^{(p-1-\gamma) /(p-1-\alpha)}, \\
\text { if } \beta=p \\
\frac{1}{(1+x)^{(\beta-p)(p-1-\gamma) /((p-1)(p-1-\alpha))}} \\
\times(L(1+x))^{(p-1-\gamma) /((p-1)(p-1-\alpha))} \\
\text { if } p<\beta<\frac{(\lambda+1)(p-1-\alpha)+(\alpha-\gamma) p}{p-1-\gamma} \\
\text { if } \beta>\frac{(\lambda+1)(p-1-\alpha)+(\alpha-\gamma) p}{p-1-\gamma}, \\
\frac{1}{(1+x)^{(\lambda-p+1) /(p-1)}} \\
\times\left(\int_{1}^{x+2} \frac{L(s)}{s} d s\right) \\
\text { if } \left.\beta=\frac{(\lambda+1)(p-1-\alpha)+(p-1)}{p-1-\gamma}, \gamma\right) p
\end{array}\right.
\end{aligned}
$$


Consequently, we deduce that (63) has a unique solution $u$ satisfying

$$
u(x) \approx\left\{\begin{array}{l}
\left(\int_{1+x}^{\infty} \frac{(L(s))^{1 /(p-1)}}{s} d s\right)^{(p-1) /(p-1-\alpha)} \\
\text { if } \beta=p, \\
(1+x)^{(p-\beta) /(p-1-\alpha)}(L(1+x))^{1 /(p-1-\alpha)}, \\
\text { if } p<\beta<\frac{(\lambda+1)(p-1-\alpha)+(\alpha-\gamma) p}{p-1-\gamma}, \\
(1+x)^{(p-1-\lambda) /(p-1-\gamma)}, \\
\text { if } \beta>\frac{(\lambda+1)(p-1-\alpha)+(\alpha-\gamma) p}{p-1-\gamma} \\
(1+x)^{(p-1-\lambda) /(p-1-\gamma)}\left(\int_{1-x}^{\eta} \frac{L(s)}{s} d s\right)^{1 /(p-1-\alpha)} \\
\text { if } \beta=\frac{(\lambda+1)(p-1-\alpha)+(\alpha-\gamma) p}{p-1-\gamma} .
\end{array}\right.
$$

\section{References}

[1] B. Acciaio and P. Pucci, "Existence of radial solutions for quasilinear elliptic equations with singular nonlinearities," Advanced Nonlinear Studies, vol. 3, no. 4, pp. 511-539, 2003.

[2] R. P. Agarwal, H. Lü, and D. O'Regan, "Existence theorems for the one-dimensional singular $p$-Laplacian equation with sign changing nonlinearities," Applied Mathematics and Computation, vol. 143, no. 1, pp. 15-38, 2003.

[3] R. P. Agarwal, H. Lü, and D. O'Regan, “An upper and lower solution method for the one-dimensional singular $p$ Laplacian," Georgian Academy of Sciences A, vol. 28, pp. 13-31, 2003.

[4] R. P. Agarwal, H. Lü, and D. O’Regan, "Eigenvalues and the onedimensional p-Laplacian," Journal of Mathematical Analysis and Applications, vol. 266, no. 2, pp. 383-400, 2002.

[5] I. Bachar, S. Ben Othman, and H. Mâagli, "Existence results of positive solutions for the radial $p$-Laplacian," Nonlinear Studies, vol. 15, no. 2, pp. 177-189, 2008.

[6] I. Bachar, S. Ben Othman, and H. Mâagli, "Radial solutions for the $p$-Laplacian equation," Nonlinear Analysis: Theory, Methods \& Applications, vol. 70, no. 6, pp. 2198-2205, 2009.

[7] E. Calzolari, R. Filippucci, and P. Pucci, "Existence of radial solutions for the $p$-Laplacian elliptic equations with weights," American Institute of Mathematical Sciences Journal, vol. 15, no. 2, pp. 447-479, 2006.

[8] D.-P. Covei, "Existence and asymptotic behavior of positive solution to a quasilinear elliptic problem in $\mathbb{R}^{N}$," Nonlinear Analysis: Theory, Methods \& Applications, vol. 69, no. 8, pp. 2615-2622, 2008.

[9] M. Ghergu and V. D. Rădulescu, Nonlinear PDEs Mathematical Models in Biology, Chemistry and Population Genetics, Springer Monographs in Mathematics, Springer, Heidelberg, Germany, 2012.

[10] J. V. Goncalves and C. A. P. Santos, "Positive solutions for a class of quasilinear singular equations," Electronic Journal of Differential Equations, vol. 2004, no. 56, pp. 1-15, 2004.

[11] D. D. Hai and R. Shivaji, "Existence and uniqueness for a class of quasilinear elliptic boundary value problems," Journal of Differential Equations, vol. 193, no. 2, pp. 500-510, 2003.
[12] X. He and W. Ge, "Twin positive solutions for the onedimensional $p$-Laplacian boundary value problems," Nonlinear Analysis: Theory, Methods \& Applications, vol. 56, no. 7, pp. 975-984, 2004.

[13] M. Karls and A. Mohammed, "Integrability of blow-up solutions to some non-linear differential equations," Electronic Journal of Differential Equations, vol. 2004, pp. 1-8, 2004.

[14] C.-G. Kim and Y.-H. Lee, "Existence of multiple positive radial solutions for $p$-Laplacian problems with an $L^{1}$-indefinite weight," Taiwanese Journal of Mathematics, vol. 15, no. 2, pp. 723-736, 2011.

[15] P. Pucci, M. García-Huidobro, R. Manásevich, and J. Serrin, "Qualitative properties of ground states for singular elliptic equations with weights," Annali di Matematica Pura ed Applicata IV, vol. 185, pp. S205-S243, 2006.

[16] H. Brezis and S. Kamin, "Sublinear elliptic equations in $\mathbb{R}^{n}$," Manuscripta Mathematica, vol. 74, no. 1, pp. 87-106, 1992.

[17] R. Chemmam, A. Dhifli, and H. Mâagli, "Asymptotic behavior of ground state solutions for sublinear and singular nonlinear Dirichlet problems," Electronic Journal of Differential Equations, vol. 2011, no. 88, pp. 1-12, 2011.

[18] A. L. Edelson, "Entire solutions of singular elliptic equations," Journal of Mathematical Analysis and Applications, vol. 139, no. 2, pp. 523-532, 1989.

[19] M. Ghergu and V. Rădulescu, "Ground state solutions for the singular Lane-Emden-Fowler equation with sublinear convection term," Journal of Mathematical Analysis and Applications, vol. 333, no. 1, pp. 265-273, 2007.

[20] A. V. Lair and A. W. Shaker, "Classical and weak solutions of a singular semilinear elliptic problem," Journal of Mathematical Analysis and Applications, vol. 211, no. 2, pp. 371-385, 1997.

[21] A. Mohammed, "Ground state solutions for singular semilinear elliptic equations," Nonlinear Analysis: Theory, Methods \& Applications, vol. 71, no. 3-4, pp. 1276-1280, 2009.

[22] C. A. Santos, "On ground state solutions for singular and semi-linear problems including super-linear terms at infinity," Nonlinear Analysis: Theory, Methods \& Applications, vol. 71, no. 12, pp. 6038-6043, 2009.

[23] E. Seneta, Regularly Varying Functions, vol. 508 of Lecture Notes in Mathematics, Springer, Berlin, Germany, 1976.

[24] J. Trubek, "Asymptotic behavior of solutions to $\Delta u+K u^{\sigma}=0$ on $\mathbb{R}^{n}$ for $n \geq 3$," Proceedings of the American Mathematical Society, vol. 106, no. 4, pp. 953-959, 1989. 


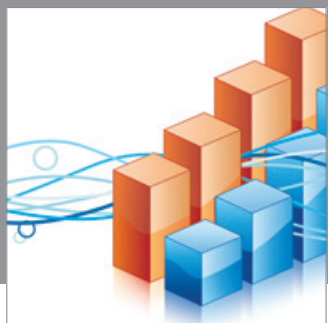

Advances in

Operations Research

mansans

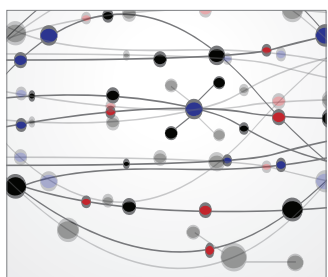

The Scientific World Journal
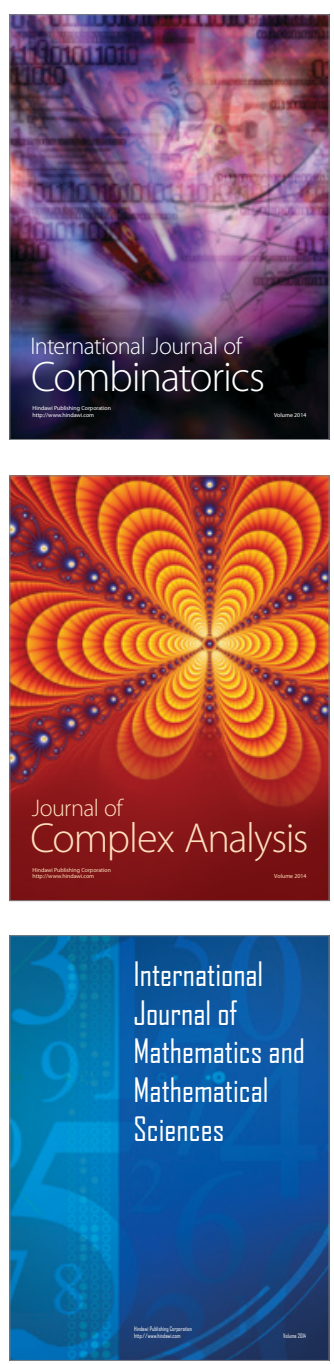
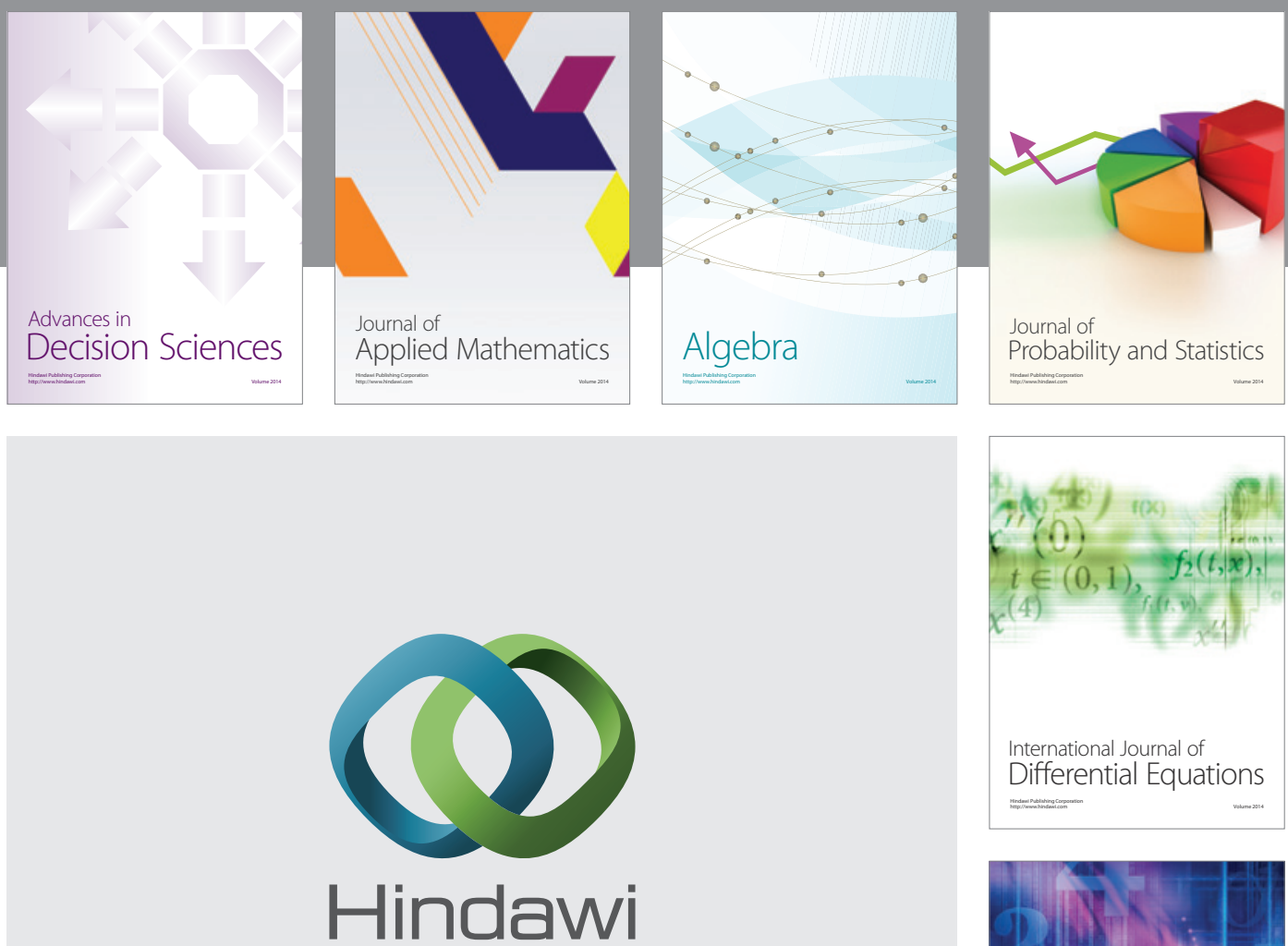

Submit your manuscripts at http://www.hindawi.com
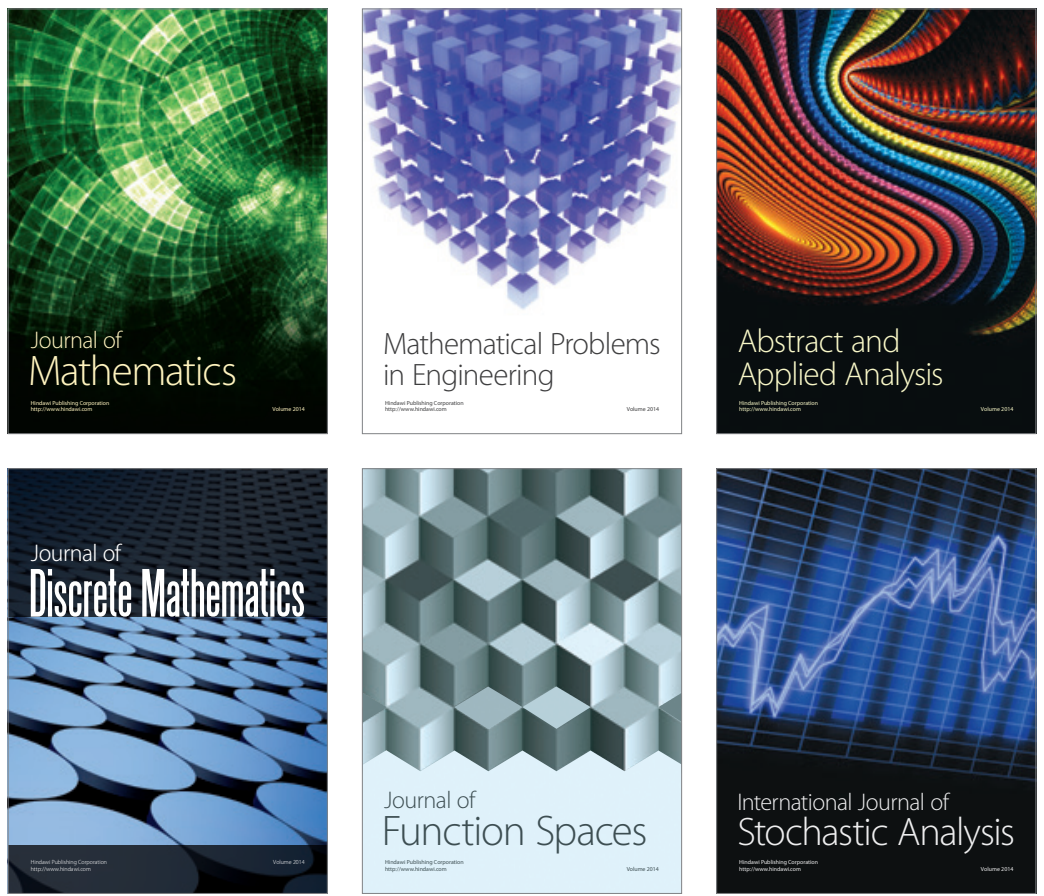

Journal of

Function Spaces

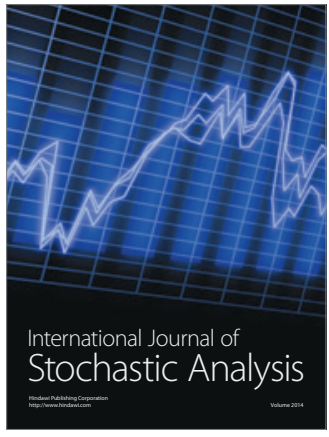

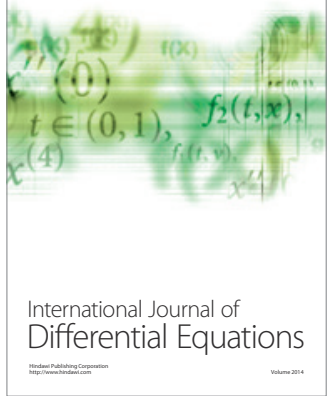
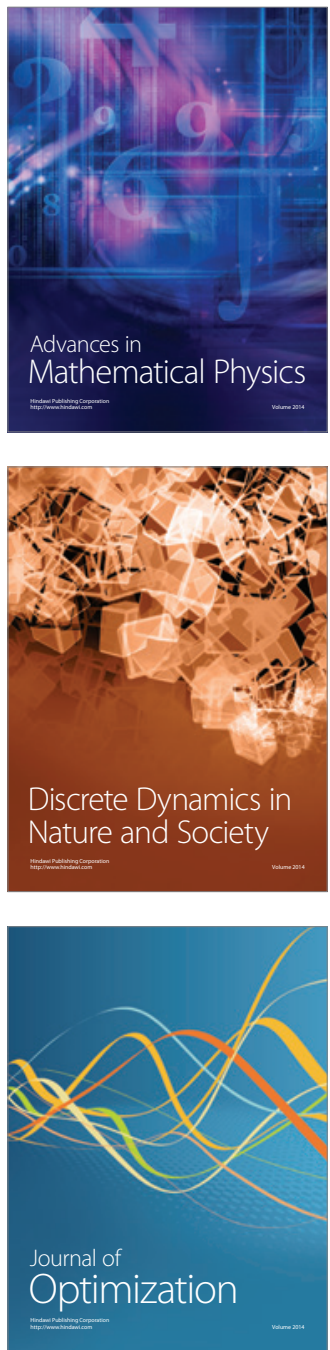